\title{
"A gente sente que precisa e pode...": os desafios para a inclusão da saúde mental na Atenção Básica
}

\author{
"We feel that we need to and we can...": challenges for the inclusion of mental health care in \\ primary health care \\ "Sentimos que necesitamos y podemos ...": los desafíos de la inclusión de la salud mental en la \\ atención primaria
}

Carina Robles Angelini. Universidade Estadual de Campinas (UNICAMP). Campinas, SP, Brasil. carinaangelini@gmail.com (Autora correspondente) Maria do Carmo Gullaci Guimarães Caccia-Bava. (Caccia-Bava MCG). Universidade de São Paulo. Departamento de Medicina Social. Faculdade de Medicina de Ribeirão Preto, SP, Brasil. mcbava@fmrp.usp.br

\section{Resumo}

A política de saúde mental brasileira enfatiza a necessidade de inclusão do cuidado da saúde mental desde o nível primário de atenção à saúde, em uma rede diversificada de cuidado coordenado e humanizado. Objetivos: compreender como os trabalhadores das equipes de saúde da família dimensionam e organizam suas práticas de cuidado ao doente mental. Métodos: estudo qualitativo em que foi utilizado grupo focal como método de coleta e o Construcionismo Social como referencial teórico para análise. Resultados: embora reconheçam a dificuldade de mudar o foco de cuidado da doença para a pessoa, os profissionais percebem a necessidade de mudança e as potencialidades da ESF ressaltando que "há algo mais que se pode fazer". Conclusão: as potencialidades da ESF podem ser ativadas com investimentos nas interações interpessoais e na construção de novos saberes, e os processos de mudança devem ser disparados por toda a equipe além do NASF e das equipes de saúde mental.

\begin{abstract}
The Brazilian mental health policy emphasizes the need for mental health care to be included in all levels of health care, from primary to tertiary, including emergency care and therapeutic residences. This policy has guided the construction of a diverse network of coordinated and humanized assistance for patients with mental illness. Objectives: the objective of this study is to understand how family health team workers position themselves and organize their practices in regard to mental health care. Methods: focus group sessions with a family health team were used to collect qualitative data, and social constructionism was used as the theoretical framework for analysis. Results: professionals recognize that there is a need for a change of focus from the disease to the person, but this seems to be difficult to achieve. However, professionals recognize the potentiality of this need and that "there is something else that I can do". Conclusion: the potential of the ESF can be activated through investment in interpersonal interactions and the construction of new knowledge, and the processes of change should be triggered by the whole team, rather than just the NASF and mental health teams.
\end{abstract}

\section{Resumen}

La política brasileña para la salud mental enfatiza la necesidad de incluir el cuidado a la salud mental desde su atención primaria hasta la terciaria en una red diversa de asistencia coordinada hacia pacientes con enfermedades mentales. Objetivos: conocer el discurso de los trabajadores de equipos de la salud familiar es importante para entender cómo se posicionan y organizan sus prácticas en el cuidado a los enfermos mentales. Métodos: estudio cualitativo mediante sesión de grupo focal con un equipo de salud de la familia y con el construccionismo social como un marco teórico para el análisis. Resultados: aunque se reconozca la necesidad de cambio del cuidado de la enfermedad para la persona, los profesionales reconocen la potencialidad de la ESF, resaltando que "hay algo más que se puede hacer". Conclusión: el potencial de la ESF se puede activar con investimentos en las relaciones interpersonales y en la construcción de nuevos conocimientos, y los procesos de cambio deben ser disparados por todo el equipo, además del NASF y de los equipos de salud mental.

Como citar: Angelini CR, Caccia-Bava MCG. "A gente sente que precisa e pode...”: os desafios para a inclusão da saúde mental na Atenção Básica. 2015;10(36)1-9.

http://dx.doi.org/10.5712/rbmfc10(36)1033
Palavras-chave:

Saúde Mental

Estratégia Saúde da Família

Atenção Primária à Saúde

Keywords:

Mental Health

Family Health Strategy

Primary Health Care

Palabras clave:

Salud Mental

Estrategia de Salud Familiar

Atención Primaria de Salud

Fonte de financiamento:

declaram não haver.

Parecer CEP:

8388/2006 (HCPRP), aprovado

em 09/10/2006.

Conflito de interesses:

declaram não haver.

Procedência e revisão por pares:

revisado por pares.

Recebido em: 29/10/2014.

Aprovado em: 15/07/2015. 


\section{Introdução}

A reformulação do modelo brasileiro de assistência em saúde mental teve início há mais de 30 anos, mas seus desdobramentos são atuais e refletem na política de saúde da Atenção Básica e da Saúde Mental. O Ministério da Saúde ofereceu o suporte legislativo para a implantação da Rede de Atenção Psicossocial (RAPS) ${ }^{1}$ e uma das diretrizes para a constituição dessa rede é a inclusão do cuidado da saúde mental desde o nível primário de atenção até o especializado, incluindo atenção às urgências e a implantação de vários dispositivos de desinstitucionalização.

Nesse sentido, pontos estruturantes da política nacional de atenção básica ${ }^{2}$ são convergentes com os pressupostos da desinstitucionalização preconizados pela reforma psiquiátrica. Ambos reconhecem a complexidade do processo saúde-doença com seus elementos complementares e indissociáveis que envolvem o corpo, o contexto sociocultural, a capacidade das pessoas de lidarem com as adversidades da vida, o grau de participação social e o exercício de cidadania. ${ }^{3}$

Essas concepções apontam para a complexidade das demandas que a Atenção Básica deve lidar e por isso é necessário o investimento em espaços de diálogos que ampliem a responsabilização das equipes em práticas de saúde diferentes das tradicionais. Caso contrário, práticas automatizadas podem dificultar a implantação de ações de fortalecimento da atenção à saúde mental na Atenção Básica. ${ }^{4}$

Anterior à formulação da RAPS e com o objetivo de qualificar e ampliar a resolutividade das ESF, em 2008 foi adotada a estratégia do apoio matricial e formalizada a criação dos Núcleos de Apoio à Saúde da Família (NASF). O apoio matricial deve oferecer suporte técnico para ampliação das ações das equipes e favorecer mudanças na atitude e na atuação dos profissionais, incluindo ações interssetoriais e interdisciplinares. ${ }^{5}$ No entanto, uma das limitações para a ação das equipes matriciais é a participação e o envolvimento dos trabalhadores das unidades de saúde, seja pela organização do processo de trabalho ou pela concepção de cuidado. ${ }^{6}$

A presença de equipes como NASF, a proximidade entre profissionais da saúde mental e da ESF, assim como a intensidade das articulações entre as equipes podem favorecer mudanças nas formas de abordagem dos transtornos mentais ao longo do tempo. ${ }^{7}$

O presente artigo é derivado de uma dissertação de mestrado e teve como objetivo acessar os sentidos construídos pelos profissionais de saúde da ESF sobre o cuidado em saúde mental de maneira a identificar os desafios para a implementação de ações em saúde mental na Atenção Básica.

\section{Método: a construção e análise do material}

Foi realizado um estudo qualitativo exploratório com uma equipe de saúde da família em um município do interior do Estado de São Paulo. Inicialmente, procedeu-se à aproximação com a unidade de saúde e com a equipe para conhecer a estrutura física, o fluxo de atendimento e também o território no qual estava inserida. Para estas atividades, foram realizadas quatro visitas à unidade de ESF.

O grupo focal foi a técnica de coleta dos dados escolhida, pois fornece dados de natureza qualitativa e prioriza os contatos horizontais entre os participantes. ${ }^{8}$ Ocorreu em sessão única, na própria unidade de saúde, na sala de reuniões e durou 1 hora e 20 minutos. O estudo contou com uma 
amostra proposital: a equipe que mais encaminhou pacientes ao Centro de Atenção Psicossocial (CAPS) no ano anterior. Todos da equipe aceitaram participar, totalizando 12 pessoas, além da pesquisadora que conduziu o grupo.

Pretendeu-se acessar os sentidos sobre o cuidado ao portador de transtorno mental grave e os disparadores da conversa foram introduzidos no grupo de forma dinâmica com objetivo de manter o foco da conversa e facilitar o fluxo de ideias: 1 - "o que vem à mente quando ouvem as palavras: doente mental ou louco?", 2 - "existem pessoas que consideram doentes mentais na área de abrangência da ESF?", 3 - "como é o contato da ESF com essas pessoas?", 4 - "na opinião da equipe, qual é o local e o tipo do cuidado que devem ser oferecidos ao portador de transtorno mental grave?", 5 - "quem deve ser o responsável pelo cuidado a eles?", 6 - "a equipe conhece a rede de serviços de saúde mental do município?", 7 - "quais são os recursos que a equipe possui e quais recursos não possuem para o cuidado em saúde mental?".

O estudo utilizou o Construcionismo Social como referencial teórico-metodológico, que entende a realidade como socialmente construída nas e pelas interações sociais. Nesta abordagem, o conhecimento é o produto de uma determinada época e alguns conceitos usualmente tratados como verdades absolutas podem ser relativizados. ${ }^{9}$ Ao se assumir esta concepção, considera-se o conhecimento que as pessoas têm da realidade e, abre-se para a construção de diferentes versões de uma determinada realidade. ${ }^{10}$

Para o desenvolvimento deste estudo, a linguagem foi valorizada e preocupou-se, sobretudo, em conhecer como as pessoas falam, explicam os fatos e suas experiências de trabalhadores, como se posicionam e planejam suas ações. O material de análise da pesquisa foi o registro da audiogravação do grupo focal e anotações de campo. A análise dos resultados consistiu em: 1 - transcrição verbatim e edição do material audiogravado em editor de texto; 2 - imersão nos conteúdos e atenção no processo conversacional do grupo; 3 - tematização sequencial das conversas e 4 - construção de sínteses temáticas.

Após leitura do material transcrito, foram recortados momentos do grupo que pudessem evidenciar como os participantes descreveram e se posicionaram em relação aos temas. A identificação e escolha das categorias de análise partiram do processo ativo de aproximação da pesquisadora com o material coletado.

Da leitura do conteúdo transcrito foram construídas duas categorias gerais que refletiam o objetivo do trabalho: a) o doente mental e b) o cuidado da equipe dirigido a ele. A partir dessas duas categorias, emergiram categorias temáticas que refletiam os sentidos construídos pelo grupo sobre: quem são os doentes mentais; os desafios do cuidado; o trabalho da equipe de ESF e as potencialidades da equipe.

A abordagem teórica utilizada considera que os sentidos são construídos em atividades relacionais, fruto da conversa entre as pessoas. Dessa forma, há uma interdependência entre o observador e universo observado e não é possível uma separação entre o pesquisador e o contexto. ${ }^{11}$

Este estudo foi aprovado por Comitê de Ética em Pesquisa (HCPRP no 8388/2006). Aos participantes foram esclarecidos os objetivos da pesquisa e foi respeitada a disponibilidade, a voluntariedade e interesse em participar. Para preservação do sigilo, os nomes foram trocados por nomes fictícios.

\section{Análise e discussão dos resultados}

A partir do material foram selecionados trechos da conversa grupal que, por meio do processo de tematização sequencial, possibilitaram observar os sentidos produzidos pelo grupo. 
O doente mental: conseguir "olhar com os olhos da alma"

O grupo relatou situações vivenciadas no dia-a-dia da equipe. Dentre essas, o caso do senhor Juliano que, "um dia arrancou o telhado da casa".

Carla: E ele tirou o telhado da casa porque ele já estava louco, né? Aí caiu uma chuva terrível aqui no bairro e molhou tudo que ela tinha dentro da casa [...] Foi feito Boletim de Ocorrência... Então, está gerando uma série de problemas [...] Podia morrer logo.

Os repertórios interpretativos iniciais utilizados pelo grupo deram sentido ao doente mental como aquele que possui comportamentos agressivos, reações imprevisíveis e constrangedoras. Ao falarem sobre o convívio social do doente mental, os estereótipos da incapacidade e da periculosidade apareceram. Esse dado não difere do que observaram outros autores ${ }^{4,12}$ e a permanência destes pode impedir a superação da situação de segregação e isolamento do doente mental.

A fala de Carla foi o ponto de partida para que relatos de casos difíceis ocorressem e a doença, os sintomas e os diagnósticos foram a referência para compreender os pacientes e justificar suas condutas. Isso reflete o quanto a perspectiva do modelo biomédico consolida modelos explicativos de determinadas doenças. ${ }^{13}$

Carla: Esse senhor, ele é esquizofrênico, né, doutora?

Adriana: [...] eu só tinha visto ele como hipertenso, mas não com o transtorno mental.

O olhar que colocou o paciente em uma condição fixa de hipertenso excluiu a possibilidade de que o mesmo pudesse adoecer mentalmente. Isso pode ocorrer porque, tradicionalmente, as intervenções baseadas em práticas nosológicas consideram somente "um" problema ${ }^{14}$ e quando várias deficiências ou desvantagens impedem o diagnóstico único de um paciente e dificultam a inclusão dele em um programa específico de saúde, o manejo fica mais difícil, como relatado abaixo:

Ana: [...] é um transtorno mental, mas ela é depressiva, tem síndrome do pânico, tem um monte de coisa junta, diabética, hipertensa, blá, blá, blá, sabe?

À medida que o grupo conversava sobre os casos, despontava o reconhecimento da complexidade do cuidado na Atenção Básica e os desafios colocados no dia-a-dia. Cuidar das pessoas em seus contextos de vida é altamente complexo ${ }^{15}$ e exige adequações constantes e projetos terapêuticos singulares. Por outro lado, a multiplicidade dos vínculos e dos acontecimentos nos espaços onde as pessoas vivem podem ser exploradas e podem gerar uma produção de cuidado mais comprometida com os sujeitos de forma mais integral. ${ }^{16}$

Dessa forma, olhar para a pessoa e para seu sofrimento e reconhecer a complexidade e a necessidade de cuidado pode favorecer os vínculos entre pacientes e equipes. Além disso, o sofrimento pode ser objeto da Atenção Primária, principalmente quando se trata do manejo de condições crônicas de saúde. ${ }^{17}$ Nesse sentido, é tão importante para a equipe de saúde saber como a pessoa vive, sofre e que recurso utiliza para lidar com seu adoecimento, quanto o diagnóstico para se definirem as condutas.

Nesse sentido, a conversa fluida do grupo promoveu sentidos sobre a possibilidade de "olharem com os olhos da alma" e reconhecerem novas formas de cuidado no contexto comunitário: 
Lia: Olhar com os olhos da alma, né? [...] porque, assim, às vezes você começa a conversar com uma pessoa, [...] que tem essas dificuldades, e aí você vai prestando atenção em algumas coisas e vai se comunicando de um jeito que você entende [...] você vê que é uma pessoa que sofre.

Lia era uma Agente Comunitária de Saúde e foi por meio de sua fala que surgiu no grupo um olhar diferente que pode estimular uma atuação contínua e personalizada de cuidado, pois reflete a transformação da concepção de saúde não como ausência de doença, mas como possibilidades de vida. Quando o foco de cuidado fica centrado na doença, a distância entre os profissionais de saúde e a pessoa que adoece inviabiliza a compreensão e invenção de novas formas de trocas e diálogos. ${ }^{18}$

As versões sobre o cuidado: "fica assim, de mãos atadas"

Enquanto circulava a ideia de que o doente mental era uma pessoa perigosa, foram expressos sentimentos de medo, de impotência e de incapacidade e a angústia de não saber o que fazer.

Ana: Angústia

Patrícia: Eu acho que é angústia mesmo... você não sabe nem como conversar com a pessoa desse jeito $[\ldots]$.

Lia: Eu tenho um sentimento de incapacidade. [...] É uma coisa muito ruim... é não saber o que fazer, não saber como tratar, não saber [...] Você fica assim de mãos atadas, mesmo.

Ana: Não, é essa sensação que ela falou de incapacidade, de impotência, né?

Adriana: (...) a gente não sabe realmente abordar, [...] Realmente falta um preparo mesmo. Eu acho que a gente precisa de preparo.

Márcia: ... de medo, tem hora.

O reconhecimento da lacuna entre a necessidade das pessoas e as ferramentas de cuidado disponíveis pode ocorrer quando se está fixado em práticas restritas à formação tradicional de cada profissão. Elas são importantes, mas a abertura para práticas colaborativas, o "fazer junto" pode favorecer a criatividade para o manejo dos casos difíceis. ${ }^{19,20}$ Além disso, as articulações em busca de contatos que façam sentido para cada família/paciente são fundamentais e podem promover ao mesmo tempo a cidadania e a saúde. ${ }^{21}$

O Processo de Trabalho: "a gente sente que precisa e pode, mas não dá"

A compreensão de que a organização e uso do tempo e do espaço favorecem a gestão do cuidado na atenção básica, apontou para as possibilidades da equipe. O desejo de cuidar, mas "não saberem por onde começar", foram relatados.

Adriana: eu acho que o maior problema aqui é quando a gente tem que trabalhar o paciente que tem um transtorno grave igual o dele. Eu acho que é justamente a gente conseguir estabelecer um vínculo. Porque é que nem a Renata falou: "Ele fica no mundo dele", [...] e a gente mesmo não consegue entrar no mundo dele e trazer ele para a realidade.

Aaproximação entre paciente e equipe fica atemorizante quando os profissionais se sentem incapazes de curá-lo e parece haver uma tendência que privilegia ações pontuais de alívio dos sintomas agudos na tentativa de resolver um problema crônico. ${ }^{22}$ Isso coloca a doença e, não o sujeito, em evidência e favorece 
a cristalização das ações de cuidado centradas nos procedimentos e não nas pessoas. Mas a prática de cuidado pautada no princípio da integralidade deve caminhar para além de silenciar o sintoma ou tratar uma disfunção ${ }^{21}$ e isso não quer dizer que os procedimentos medicamentosos (prescrição de psicofármacos) não sejam importantes no manejo das doenças mentais.

A exploração de outras tecnologias de cuidado como grupos, atendimentos no domicílio, atividades e articulações no território favorecem a transformação do processo de trabalho da equipe ${ }^{23}$ mas sem que possam operar de um novo modo, a atitude de ouvir o outro, de ficar mais próximo perde prioridade diante da grande demanda na unidade.

Luciana: Não, a gente consegue dar mesmo uma atenção quando eles vêm aqui na unidade [...]. Agora, você ir atrás, você não consegue dar conta disso, né, porque tem outros "n" casos [...]. Você acaba ficando muito preso aqui dentro da unidade, né? [...] em função de você ficar só ali atendendo, atendendo, atendendo.

Renata: A gente sente que precisa e pode, mas não dá.

Ana: É a organização do trabalho é que tá precisando né?

Com relação a esse aspecto, não é suficiente a disponibilidade pessoal e isolada dos profissionais de saúde, mas a organização do processo de trabalho e envolvimento da equipe como um todo é fundamental.

Em relação às demandas de cuidado em saúde mental, há necessidade de adoção de práticas mais integrativas que favoreçam a escuta para além das demandas explícitas relacionadas a uma determinada doença ou problema de saúde. ${ }^{24}$

Ana: a partir do momento que você dá atenção pro paciente, ter uma escuta ampliada desse paciente. Aqui acho que o que todos nós temos é que todo mundo acolhe o paciente, não é só o acolhimento médico, mas o acolhimento da ACS na própria casa do paciente. Isso são recursos que a gente tem aqui.

Nesse sentido, o papel do agente comunitário de saúde (ACS) foi considerado como um recurso potente da equipe, pois ele tem a oportunidade de se vincular e conhecer outras necessidades que não estejam restritas a uma determinada doença. Ao fazer isso, vai além dos recursos institucionalizados e oferece uma atenção integral desenvolvendo papel fundamental no processo de cuidado na perspectiva da desinstitucionalização proposto pela política de saúde mental. Isso tudo é prática de cuidado da saúde mental, é prática de cuidado integral: ${ }^{21}$ manter o compromisso com a vida e a cidadania dos sujeitos em lugares onde é possível o resgate de sua autonomia e a reconstrução de sua vida e de sua história. ${ }^{22}$

As potencialidades da equipe: "há algo mais que eu posso fazer"

Ana trouxe para perto o desejo de fazer algo diferente e valorizar ações de mudança a partir da exploração dos recursos já disponíveis na equipe, mas que às vezes não são explorados.

Ana: eu fico me perguntando... a gente precisa melhorar, é bom ter essa troca. [...] A gente tem esse movimento entre nós. Então esse movimento que eu fico pensando: "Será que não precisa melhorar mais? Será que mais eu posso fazer? Eu acho que é isso que precisa com o paciente de saúde mental também, né? Não sei, da minha parte, assim, eu acho que eu poderia fazer mais. 
Renata: A sensação é essa, quando a gente sente que faz um pouquinho, nossa!. [...] mas não é assim que acontece, né?

Débora: Você tem até a sensação de tudo que você fez certo deu errado naquele dia.

Renata: Isso! Aí você pega o carro para ir embora e vai pensando, entendeu? Além de tudo isso que falaram, eu às vezes sinto até uma carga assim, um negócio. Me sinto também incompetente, sabe? Não sei me comunicar, mas acho que é isso!

A dificuldade de comunicação expressa pela participante marca a intensidade afetiva dos relatos do grupo e a forma dos profissionais e equipe lidarem com os sentimentos pode ser a ferramenta facilitadora ou obstrutora do cuidado. A necessidade de ajuda para que a equipe possa fazer o cuidado de um jeito diferente e se sentir potente ficou evidente. Foi apontada a falta de apoio de uma equipe de saúde mental no cotidiano, de capacitação e de espaços de trocas.

Nesse sentido, é importante reconhecer o limite da atenção básica quando são necessárias intervenções psiquiátricas especializadas em momentos pontuais de crises. Afinal, a desinstitucionalização não pode significar desassistência psiquiátrica ${ }^{25} \mathrm{e}$, em alguns casos, é necessário proximidade de uma equipe que auxilie no desenvolvimento de intervenções específicas, de forma próxima e intensiva. ${ }^{7}$

No trecho abaixo, as falas apontam para as potencialidades do trabalho conjunto como um recurso que fortalece e motiva as ações da equipe.

Ana: [...] a gente se abre e se coloca a novos conhecimentos. Quando a gente vive a nossa dificuldade, né, a gente ter alguém.... Então eu acho que colocando a gente vai encontrando as respostas. Eu acho que a gente podia fazer sempre isso [...] Eu me sinto sempre respaldada com esse trabalho de equipe. [...] Trabalhar assim é ótimo, né! É o que eu quero na vida (risos).

Foi valorizada a presença de toda a equipe, as discussões de caso e o planejamento de ações. Nesse sentido, as propostas que sustentam o trabalho de articulação entre profissionais da saúde mental e atenção básica, seja das equipes de saúde mental ou do NASF, são desafiadoras. Não se trata de incluir profissionais especialistas na Atenção Básica, mas o desafio é construir e fazer juntos, planejando ações que alinhem o processo de trabalho e que favoreça que todos da equipe sejam valorizados. ${ }^{5}$

\section{Considerações finais}

Embora atualmente se reconheça a necessidade de apoio e formação permanente das Equipes de Saúde da Família e da importância dos NASFs, a implementação do cuidado da saúde mental na Atenção Básica é tarefa desafiadora e este estudo pode respaldá-las. Além disso, algumas das potencialidades encontradas pelos profissionais participantes podem apontar caminhos para a superação de dificuldades.

Todavia, há que se considerar limitações do estudo relacionadas ao desenho do mesmo. Por ser um estudo qualitativo e com uma equipe de saúde apenas, trata-se de uma investigação em um contexto e em uma realidade definida e os resultados devem ser utilizados com parcimônia, pois trazem recortes de encontros pontuais e com uma equipe de saúde da família também delimitada. Muito embora outros autores tenham observados resultados semelhantes, ${ }^{4}$ os achados não são generalizáveis para outros contextos, mas podem subsidiar outros estudos exploratórios sobre o tema. 
Outra limitação do estudo é o fato da pesquisadora, na ocasião da coleta de dados, ser uma psicóloga e trabalhadora de um serviço de saúde mental (CAPS), que mesmo não se posicionando como expert no assunto discutido pelo grupo, foi colocada no lugar de interlocutora privilegiada que pudesse ouvir as necessidades da equipe. ${ }^{9}$ Nesse sentido, a pesquisadora estava incluída no grupo e sua presença mudava o contexto da equipe e convidava o grupo para falar sobre sentimentos, desejos e medos em relação ao cuidado do doente mental.

Os resultados desse estudo, contudo, refletem alguns dos desafios para a inserção do cuidado à saúde mental na Atenção Básica. A mudança do modelo de atenção, em especial ao doente mental, é um grande desafio para as políticas públicas sobre o tema. Há reconhecimento por parte dos profissionais da necessidade de mudança, mas parece ser difícil mudar o foco de cuidado da doença para a pessoa e assim as ações, quando acontecem, são isoladas e desarticuladas. ${ }^{4}$

Com isso, sentimentos de medo, impotência e incapacidade podem ser paralisantes para a não exploração de outras tecnologias de cuidado. Todavia, os profissionais reconhecem que "há algo mais que se possa fazer", como as ações desenvolvidas pelo agente comunitário de saúde na vinculação e conhecimento das necessidades dos pacientes. Essas potencialidades podem ser ativadas com investimentos nas interações interpessoais e na construção de novos saberes. Para tanto, é necessário que as responsabilidades nos processos de mudança sejam assumidas por toda a equipe e o papel do NASF ou das equipes de saúde mental ganha destaque na estimulação de diálogos horizontalizados e permanentes com atenção especial ao processo de trabalho das equipes.

\section{Referências}

1. Brasil. Ministério da Saúde. Portaria no 3.088 de 23 de Dezembro de 2011. Brasília: Ministério da Saúde; 2011.

2. Brasil. Ministério da Saúde. Portaria no 2488 de 21 de outubro de 2011. Brasília: Ministério da Saúde; 2011.

3. Figueiredo MD, Campos RO. Saúde Mental na atenção básica à saúde de Campinas, SP: uma rede ou um emaranhado? Ciên Saúde Coletiva. 2009;14(1):129-38. DOI: http://dx.doi.org/10.1590/S1413-81232009000100018

4. Neves R, Dimenstein M, Paulon S, Nardi H, Bravo O, Galvão V, et al. A saúde mental no sistema único de saúde do Brasil: duas realidades em análise. Av Psicol Latinoam. 2012;30(2):356-68.

5. Brasil. Ministério da Saúde. Diretrizes do NASF. Núcleo de Apoio à Saúde da Família. Brasília: Ministério da Saúde;2009.

6. Morais APP, Tanaka O. Apoio matricial em saúde mental: alcances e limites na atenção básica. Saude Soc. 2012; 21(1):161-70. DOI: http://dx.doi.org/10.1590/S0104-12902012000100016

7. Binotto AL, Santos LL, Lourosa QL, Sant'Anna SC, Zanetti ACG, Forster AC, et al. Interface saúde da família \& saúde mental: uma estratégia para o cuidado. Rev Bras Med Fam Comunidade. 2012;7(23):83-9. DOI: http://dx.doi.org/10.5712/ rbmfc7(23)132

8. Trad LAB. Grupos focais: conceitos, procedimentos e reflexões baseadas em experiências com o uso da técnica em pesquisas de saúde. Physis. 2009;19(3):777-96. DOI: http://dx.doi.org/10.1590/\$0103-73312009000300013

9. Guanaes C. A construção da mudança em terapia de grupo: um enfoque construcionista social. São Paulo: Vetor; 2006.

10. Spink MJP, Frezza RM. Práticas discursivas e produção de sentidos: a perspectiva da Psicologia Social. In: Spink MJP, org. Práticas discursivas e produção de sentidos no cotidiano. 2a ed. São Paulo: Cortez; 2000.

11. Grandesso MA. O individual e o social: em busca da complexidade. In: Grandesso MA. Sobre a reconstrução do significado: uma análise epistemológica e hermenêutica da prática clínica. São Paulo: Casa do Psicólogo; 2000. p.104-16.

12. Boarini ML, Quijo I. A Reforma psiquiátrica e as andorinhas. Rev Dep Psicol. 2007;19(2):369-379. 
13. Vasconcelos I, Lins SLB, Vargas GC, Ribeiro KCS. Concepções loucura um traçado histórico-cultural: uma com o construcionismo social. Mental. 2010;8(14):49-63.

14. Alves D. Integralidade nas políticas de saúde mental. In: Pinheiros R, Mattos RA, eds. Os sentidos da integralidade na atenção e no cuidado à saúde. Rio de Janeiro: Abrasco; 2001. p.167-76.

15. Lancetti A. Clínica peripatética. São Paulo: Hucitec; 2006.

16. Rodrigues ES, Moreira MIB. A interlocução da saúde mental com atenção básica no município de Vitoria/ES. Saúde Soc. 2012;21(3):599-611. DOI: http://dx.doi.org/10.1590/S0104-12902012000300007

17. Brasil. Organização Mundial da Saúde. Cuidados Inovadores para condições crônicas: componentes estruturais de ação, relatório mundial. Brasília; 2003.

18. Kinoshita R. Contratualidade e reabilitação psicossocial. In: Pitta A, ed. Reabilitação psicossocial no Brasil. São Paulo: Hucitec; 2001. p.55-9.

19. Correia VR, Barros S, Colvero LA. Saúde mental na atenção básica: prática da equipe de saúde da família. Rev Esc Enferm USP. 2011;45(6):1501-6. DOI: http://dx.doi.org/10.1590/S0080-62342011000600032

20. Mielke FB, Olchowsky A. Saúde mental na estratégia de saúde da família: a avaliação de apoio matricial. Rev Bras Enferm. 2012;63(6):900-7. DOI: http://dx.doi.org/10.1590/S0034-71672010000600005

21. Mattos R. Os sentidos da integralidade: algumas reflexões acerca de valores que merecem ser definidos. In: Pineiros R, Mattos R, eds. Os sentidos da integralidade na atenção e no cuidado à saúde. Rio de Janeiro: Abrasco; 2001. p.39-64.

22. Feuerwerker LCM, Merhy EE. A contribuição da atenção domiciliar para a configuração de redes substitutivas de saúde: desinstitucionalização e transformação de práticas. Rev Panam Salud Publica. 2008;24(3):180-8. DOI: http://dx.doi. org/10.1590/S1020-49892008000900004

23. Camuri D, Dimenstein M. Processos de trabalho em saúde: práticas de cuidado em saúde mental na estratégia saúde da família. Saúde Soc. 2010;19(4):803-13. DOI: http://dx.doi.org/10.1590/S0104-12902010000400008

24. Mielke FB, Olchowsky A. As ações de saúde mental na estratégia de saúde da família e as tecnologias em saúde. Esc Anna Nery. 2011;15(4):762-8. DOI: http://dx.doi.org/10.1590/S1414-81452011000400015

25. Vecchia MD, Martins STF. Desinstitucionalização dos cuidados a pessoas com transtornos mentais na atenção básica: aportes para a implementação de ações. Interface Comun Saúde Educ. 2009;13(28):151-64. 